ALEA, Lat. Am. J. Probab. Math. Stat. 15, 1293-1309 (2018)

DOI: $10.30757 /$ ALEA.v15-48

\title{
Reversal property of the Brownian tree
}

\section{Romain Abraham and Jean-François Delmas}

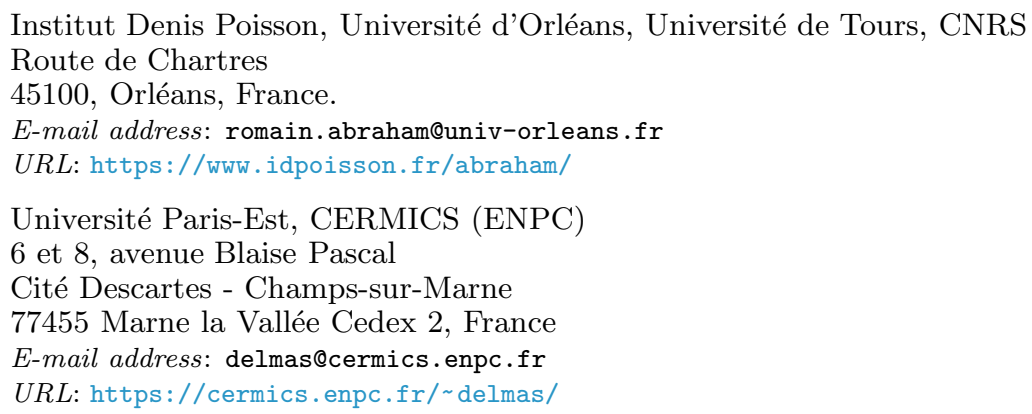

Abstract. We consider the Brownian tree introduced by Aldous and the associated Q-process which consists in an infinite spine on which are grafted independent Brownian trees. We present a reversal procedure on these trees that consists in looking at the tree downward from its top: the branching points becoming leaves and leaves becoming branching points. We prove that the distribution of this tree is invariant under this reversal procedure, which provides a better understanding of previous results from Bi and Delmas (2016).

\section{Introduction}

Continuous state branching $(\mathrm{CB})$ processes are stochastic processes that can be obtained as the scaling limits of sequences of Galton-Watson processes when the initial number of individuals tends to infinity. They hence can be seen as a model for a large branching population. The genealogical structure of a CB process can be described by a forest of continuum random trees (CRT). The notion of CRT was introduced first in Aldous (1991) for the quadratic critical case, the link with CB processes can be found in Le Gall and Le Jan (1998) and Duquesne and Le Gall (2002) for the general critical and sub-critical cases, and Abraham and Delmas (2012) for the super-critical case. We shall only consider the quadratic case; it is

Received by the editors October 16th, 2017; accepted October 10th, 2018.

2010 Mathematics Subject Classification. 60J80, 60J85.

Key words and phrases. Stationary branching processes, Real trees, Genealogical trees.

Research supported by Grant ANR-14-CE25-0014 (ANR GRAAL). 
characterized by a branching mechanism $\psi_{\theta}$ :

$$
\psi_{\theta}(\lambda)=\beta \lambda^{2}+2 \beta \theta \lambda, \quad \lambda \in[0,+\infty),
$$

where $\beta>0$ and $\theta \in \mathbb{R}$. The sub-critical (resp. critical) case corresponds to $\theta>0$ (resp. $\theta=0$ ). The parameter $\beta$ can be seen as a time scaling parameter, and $\theta$ as a population size parameter.

In this model the population dies out a.s. in the critical and sub-critical cases. In order to model branching population with a stationary size distribution, which corresponds to what is observed at an ecological equilibrium, one can simply condition a sub-critical or a critical CB to not die out. This gives a Q-process, see RoellyCoppoletta and Rouault (1989) and Lambert (2007), which can also be viewed as a CB with a specific immigration. The genealogical structure of the Q-process in the stationary regime is a tree with an infinite spine. This infinite spine has to be removed if one adopts the immigration point of view, in this case the genealogical structure can be seen as a forest of trees. For $\theta>0$, let $\left(Z_{t}, t \in \mathbb{R}\right)$ be this Q-process in the stationary regime, so that $Z_{t}$ is the size of the population at time $t \in \mathbb{R}$. See Chen and Delmas (2012) for studies on this model in a more general framework. Let $A_{t}$ be the time to the most recent common ancestor of the population living at time $t$. According to Chen and Delmas (2012), we have $\mathbb{E}\left[Z_{t}\right]=1 / \theta$, and $\mathbb{E}\left[A_{t}\right]=3 / 4 \beta \theta$, so that $\theta$ is indeed a population size parameter and $\beta$ is a time parameter.

For $s<t$, let $M_{s}^{t}$ be the number of individuals at time $s$ who have descendants at time $t$. It is proven in Bi and Delmas (2016), that for fixed $\theta>0$ a time reversal property holds: in the stationary regime, the ancestor process $\left(\left(M_{s-r}^{s}, r>0\right), s \in\right.$ $\mathbb{R})$ is distributed as the descendant process $\left(\left(M_{s}^{s+r}, r>0\right), s \in \mathbb{R}\right)$, see Remark 3.14. This paper extends and explains this identity in law by reversing the genealogical tree. The idea is to see the tree as ranked branches, with each branch being attached to a longer one (the longest being the infinite spine). Then, re-attach every branch by its highest point on the same branch. Hence, branching points become leaves and leaves become branching points. Call this operation the reversal procedure. Theorem 3.9 states that, for $\theta \geq 0$, the Brownian CRT distribution is invariant by the reversal procedure and Corollary 3.13 states that, for $\theta \geq 0$, the distribution of the genealogical structure of the Q-process in the stationary regime is also invariant by the reversal procedure. See a similar result in the discrete setting of splitting trees in Dávila Felipe and Lambert (2015).

The paper is organized as follows. We first introduce in Section 2 the framework of real trees and we define the Brownian CRT that describes the genealogy of the $\mathrm{CB}$ in the quadratic case. We define in Section 3 the reversal procedure of a tree and prove the invariance property of the Brownian CRT under this reversal procedure. We then extend the result to the Brownian forest that describes the genealogy of the stationary population in the quadratic (critical and sub-critical) case.

\section{Notations}

2.1. Real trees. The study of real trees has been motivated by algebraic and geometric purposes. See in particular the survey Dress et al. (1996). It has been first used in Evans et al. (2006) to study random continuum trees, see also Evans (2008). 
Definition 2.1 (Real tree). A real tree is a metric space $\left(\mathbf{t}, d_{\mathbf{t}}\right)$ such that:

(i) For every $x, y \in \mathbf{t}$, there is a unique isometric map $f_{x, y}$ from $\left[0, d_{\mathbf{t}}(x, y)\right]$ to t such that $f_{x, y}(0)=x$ and $f_{x, y}\left(d_{\mathbf{t}}(x, y)\right)=y$.

(ii) For every $x, y \in \mathbf{t}$, if $\phi$ is a continuous injective map from $[0,1]$ to $\mathbf{t}$ such that $\phi(0)=x$ and $\phi(1)=y$, then $\phi([0,1])=f_{x, y}\left(\left[0 ; d_{\mathbf{t}}(x, y)\right]\right)$.

Notice that a real tree is a length space as defined in Burago et al. (2001). We say that a real tree is rooted if there is a distinguished vertex $\partial=\partial_{\mathbf{t}}$ which we call the root. Remark that the set $\{\partial\}$ is a rooted tree that only contains the root.

Let $\mathbf{t}$ be a compact rooted real tree and let $x, y \in \mathbf{t}$. We denote by $\llbracket x, y \rrbracket$ the range of the map $f_{x, y}$ described in Definition 2.1. We also set $\llbracket x, y \llbracket=\llbracket x, y \rrbracket \backslash$ $\{y\}$. We define the out-degree of $x$, denoted by $k_{\mathbf{t}}(x)$, as the number of connected components of $\mathbf{t} \backslash\{x\}$ that do not contain the root. If $k_{\mathbf{t}}(x)=0$, resp. $k_{\mathbf{t}}(x)>1$, then $x$ is called a leaf, resp. a branching point. We denote by $\mathcal{L}(\mathbf{t})$, resp. $\mathcal{B}(\mathbf{t})$, the set of leaves, resp. of branching points, of $\mathbf{t}$. A tree is said to be binary if the out-degree of its vertices belongs to $\{0,1,2\}$. The skeleton of the tree $\mathbf{t}$ is the set of points of $\mathbf{t}$ that are not leaves: $\operatorname{sk}(\mathbf{t})=(\mathbf{t} \backslash \mathcal{L}(\mathbf{t})) \cup\{\partial\}$. Notice that $\mathrm{cl}(\operatorname{sk}(\mathbf{t}))=\mathbf{t}$, where $\operatorname{cl}(A)$ denote the closure of $A$.

We denote by $\mathbf{t}_{x}$ the sub-tree of $\mathbf{t}$ above $x$ i.e.

$$
\mathbf{t}_{x}=\{y \in \mathbf{t}, x \in \llbracket \partial, y \rrbracket\}
$$

rooted at $x$. We say that $x$ is an ancestor of $y$, which we denote by $x \preccurlyeq y$, if $y \in \mathbf{t}_{x}$. We write $x \prec y$ if furthermore $x \neq y$. Notice that $\preccurlyeq$ is a partial order on $\mathbf{t}$. We denote by $x \wedge y$ the Most Recent Common Ancestor (MRCA) of $x$ and $y$ in $\mathbf{t}$ i.e. the unique vertex of $\mathbf{t}$ such that $\llbracket \partial, x \rrbracket \cap \llbracket \partial, y \rrbracket=\llbracket \partial, x \wedge y \rrbracket$.

We denote by $h_{\mathbf{t}}(x)=d_{\mathbf{t}}(\partial, x)$ the height of the vertex $x$ in the tree $\mathbf{t}$ and by $H(\mathbf{t})$ the height of the tree $\mathbf{t}$ :

$$
H(\mathbf{t})=\max \left\{h_{\mathbf{t}}(x), x \in \mathbf{t}\right\} .
$$

We define the set of extremal leaves of $\mathbf{t}$ by:

$$
\mathcal{L}^{*}(\mathbf{t})=\left\{y \in \mathcal{L}(\mathbf{t}), \exists x \in \mathbf{t} \text { s.t. } x \prec y \text { and } h_{\mathbf{t}_{x}}(y)=H\left(\mathbf{t}_{x}\right)\right\} .
$$

In particular, we can have $\mathcal{L}^{*}(\mathbf{t}) \neq \mathcal{L}(\mathbf{t})$, see Example 2.5.

For $\varepsilon>0$, we define the erased tree $r_{\varepsilon}(\mathbf{t})$ (sometimes called in the literature the $\varepsilon$-trimming of the tree $\mathbf{t}$ ) by

$$
r_{\varepsilon}(\mathbf{t})=\left\{x \in \mathbf{t} \backslash\{\partial\}, H\left(\mathbf{t}_{x}\right) \geq \varepsilon\right\} \cup\{\partial\} .
$$

For $\varepsilon>0, r_{\varepsilon}(\mathbf{t})$ is indeed a tree and $r_{\varepsilon}(\mathbf{t})=\{\partial\}$ for $\varepsilon>H(\mathbf{t})$. Notice that

$$
\bigcup_{\varepsilon>0} r_{\varepsilon}(\mathbf{t})=\operatorname{sk}(\mathbf{t}) \text {. }
$$

Lemma 2.2. For every compact rooted real tree $\mathbf{t}$ not reduced to the root, and every $\varepsilon \in(0, H(\mathbf{t}))>0$, the erased tree $r_{\varepsilon}(\mathbf{t})$ has finitely many leaves.

Proof: Let $\mathbf{t}$ be a compact roooted real tree not reduced to the root, and let $\varepsilon \in(0, H(\mathbf{t}))$. We set $N$ the number of leaves of $r_{\varepsilon}(\mathbf{t})$. If $N=+\infty$, there exists a (pairwise distinct) sequence $\left(y_{n}, n \in \mathbb{N}\right)$ of leaves of $r_{\varepsilon}(\mathbf{t})$. Then, by definition the subtrees $\mathbf{t}_{y_{n}}$ of $\mathbf{t}$ are pairwise disjoint and have height $\varepsilon$. Hence, if we choose for every $n \in \mathbb{N}$ a point $x_{n}$ in $\mathbf{t}_{y_{n}}$ such that $h_{\mathbf{t}_{n}}\left(x_{n}\right)=\varepsilon$, the sequence $\left(x_{n}, n \in \mathbb{N}\right)$ satisfies

$$
\forall i, j \in \mathbb{N}, i \neq j \Longrightarrow d_{\mathbf{t}}\left(x_{i}, x_{j}\right) \geq 2 \varepsilon
$$


which contradicts the compactness of the tree $\mathbf{t}$. So $N$ is finite.

We give a definition of height regularity which implies the uniqueness of the top of the tree (see Lemma 2.4 for a precise definition).

Definition 2.3 (Height regular). We say that a compact rooted real tree $\mathbf{t}$ is height-regular if, for every $\varepsilon>0$, for every $(x, y) \in \mathcal{L}\left(r_{\varepsilon}(\mathbf{t})\right)^{2} \cup \mathcal{B}\left(r_{\varepsilon}(\mathbf{t})\right)^{2}$,

$$
x \neq y \Longrightarrow h_{\mathbf{t}}(x) \neq h_{\mathbf{t}}(y) \text {. }
$$

Lemma 2.4. Let $\mathbf{t}$ be a compact height-regular tree. For every $x \in \mathbf{t}$, there exists a unique $x_{\mathbf{t}}^{*} \in \mathbf{t}_{x}$ (or simply $x^{*}$ when there is no risk of confusion) such that $h_{\mathbf{t}_{x}}\left(x_{\mathbf{t}}^{*}\right)=H\left(\mathbf{t}_{x}\right)$.

Proof: If $x \in \mathcal{L}(\mathbf{t})$, then $\mathbf{t}_{x}=\{x\}$ and the lemma holds trivially.

Let $x \in \operatorname{sk}(\mathbf{t})$. First, as $\mathbf{t}_{x}$ is compact, $H\left(\mathbf{t}_{x}\right)$ is finite and there exists at least one point $y \in \mathbf{t}_{x}$ such that $h_{\mathbf{t}_{x}}(y)=H\left(\mathbf{t}_{x}\right)$.

Assume there exists two distinct points $y, y^{\prime} \in \mathbf{t}_{x}$ such that $h_{\mathbf{t}_{x}}(y)=h_{\mathbf{t}_{x}}\left(y^{\prime}\right)=$ $H\left(\mathbf{t}_{x}\right)$. Then we have $y \wedge y^{\prime} \in \mathbf{t}_{x}$ and $h_{\mathbf{t}_{x}}\left(y \wedge y^{\prime}\right)<H\left(\mathbf{t}_{x}\right)$. We choose $\varepsilon>0$ such that $\varepsilon<H\left(\mathbf{t}_{x}\right)-h_{\mathbf{t}_{x}}\left(y \wedge y^{\prime}\right)$ and we denote by $y_{\varepsilon}$ (resp. $\left.y_{\varepsilon}^{\prime}\right)$ the unique point in $\llbracket y \wedge y^{\prime}, y \rrbracket\left(\right.$ resp. $\left.\llbracket y \wedge y^{\prime}, y^{\prime} \rrbracket\right)$ such that $d_{\mathbf{t}}\left(y_{\varepsilon}, y\right)=\varepsilon\left(\operatorname{resp} . d_{\mathbf{t}}\left(y_{\varepsilon}^{\prime}, y^{\prime}\right)=\varepsilon\right)$. Remark that these points exist by the particular choice of $\varepsilon$. Then, by definition, $y_{\varepsilon}$ and $y_{\varepsilon}^{\prime}$ are distinct leaves of $r_{\varepsilon}(\mathbf{t})$ and have the same height, which contradicts the fact that $\mathbf{t}$ is height regular.

Let $\mathbf{t}$ be a compact binary height-regular rooted real tree. For $x \in \mathbf{t}$, the vertex $x^{*}$ will be called the top of the tree $\mathbf{t}_{x}$. For such a tree, we have the equality:

$$
\mathcal{L}^{*}(\mathbf{t})=\left\{x^{*}, x \in \operatorname{sk}(\mathbf{t})\right\}
$$

By Equation (2.1), we also have

$$
\mathcal{L}^{*}(\mathbf{t})=\bigcup_{\varepsilon>0}\left\{x^{*}, x \in \mathcal{L}\left(r_{\varepsilon}(\mathbf{t})\right)\right\}
$$

and we deduce from Lemma 2.2 that if $\mathbf{t}$ is height-regular, then $\mathcal{L}^{*}(\mathbf{t})$ is at most countable.

For every $x \in \mathbf{t}$, we define the branching point of $x$ on $\llbracket \partial, \partial^{*} \rrbracket$ as

$$
\underline{x}=x \wedge \partial^{*} .
$$

For every $y \in \llbracket \partial, \partial^{*} \rrbracket$, the sub-tree (possibly reduced to its root) rooted at $y$ which does not contain neither $\partial$ nor $\partial^{*}$ is given by

$$
\tilde{\mathbf{t}}_{y}=\left\{z \in \mathbf{t}, z \wedge \partial^{*}=y\right\} .
$$

Notice that $\tilde{\mathbf{t}}_{y}$ is indeed a tree. Then, for every $x \in \mathbf{t}$, we define the maximal height of the subtree $\tilde{\mathbf{t}}_{\underline{x}}$ which is attached on $\llbracket \partial, \partial^{*} \rrbracket$ and which contains $x$ by

$$
h_{\mathbf{t}}^{\prime}(x)=H\left(\tilde{\mathbf{t}}_{\underline{x}}\right)+h_{\mathbf{t}}(\underline{x}) .
$$

See Figure 2.1 for a simplified picture of $x, \underline{x}, \mathbf{t}_{x}, x^{*}, \tilde{\mathbf{t}}_{\underline{x}}$ and $h_{\mathbf{t}}^{\prime}(x)$.

Let $\mathbf{t}$ be a compact rooted real tree and let $\left(\mathbf{t}_{i}, i \in \bar{I}\right)$ be a family of trees, and $\left(x_{i}, i \in I\right)$ a family of vertices of $\mathbf{t}$. We denote by $\mathbf{t}_{i}^{\circ}=\mathbf{t}_{i} \backslash\left\{\partial_{\mathbf{t}_{i}}\right\}$. We define the 


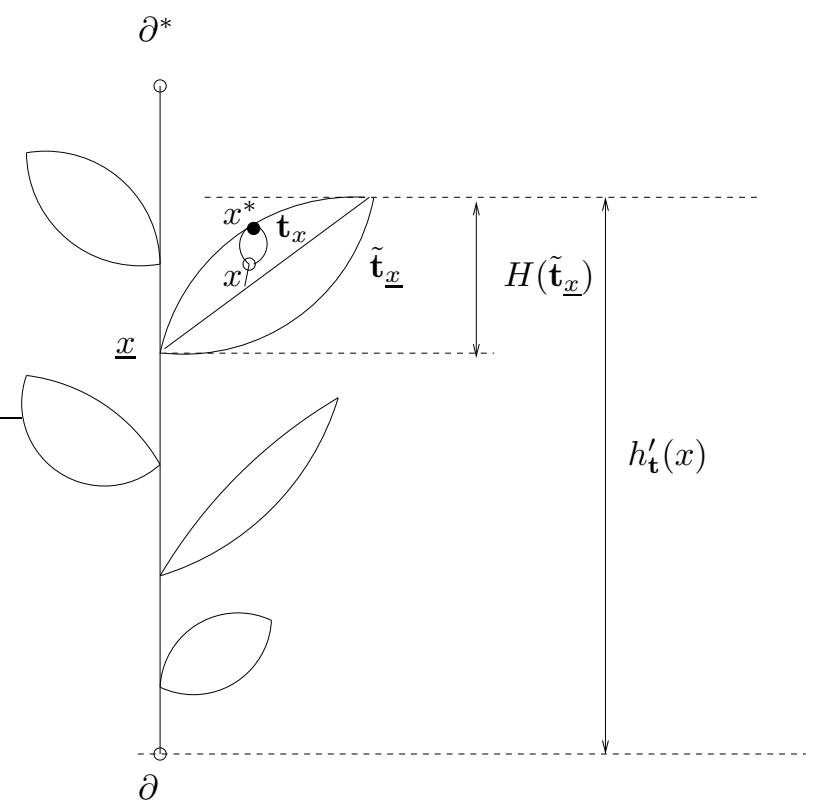

Figure 2.1. A tree $\mathbf{t}$, with $x, x_{\mathbf{t}}^{*}$ and $\underline{x}$ elements of $\mathbf{t}$, and the sub-trees $\mathbf{t}_{x}$ and $\tilde{\mathbf{t}}_{\underline{x}}$.

tree $\mathbf{t} \circledast_{i \in I}\left(\mathbf{t}_{i}, x_{i}\right)$ obtained by grafting the trees $\mathbf{t}_{i}$ on the tree $\mathbf{t}$ at points $x_{i}$ by

$$
\begin{aligned}
& \mathbf{t} \circledast_{i \in I}\left(\mathbf{t}_{i}, x_{i}\right)=\mathbf{t} \sqcup\left(\bigsqcup_{i \in I} \mathbf{t}_{i}^{\circ}\right), \\
& d_{\mathbf{t} \circledast_{i \in I}\left(\mathbf{t}_{i}, x_{i}\right)}\left(y, y^{\prime}\right) \\
& \quad= \begin{cases}d_{\mathbf{t}}\left(y, y^{\prime}\right) & \text { if } y, y^{\prime} \in \mathbf{t}, \\
d_{\mathbf{t}_{i}}\left(y, y^{\prime}\right) & \text { if } y, y^{\prime} \in \mathbf{t}_{i}^{\circ}, \\
d_{\mathbf{t}}\left(y, x_{i}\right)+d_{\mathbf{t}_{i}}\left(\partial_{\mathbf{t}_{i}}, y^{\prime}\right) & \text { if } y \in \mathbf{t} \text { and } y^{\prime} \in \mathbf{t}_{i}^{\circ}, \\
d_{\mathbf{t}_{i}}\left(y, \partial_{\mathbf{t}_{i}}\right)+d_{\mathbf{t}}\left(x_{i}, x_{j}\right)+d_{\mathbf{t}_{j}}\left(\partial_{\mathbf{t}_{j}}, y^{\prime}\right) & \text { if } y \in \mathbf{t}_{i}^{\circ} \text { and } y^{\prime} \in \mathbf{t}_{j}^{\circ} \text { with } i \neq j,\end{cases} \\
& \partial_{\mathbf{t} \circledast_{i \in I}\left(\mathbf{t}_{i}, x_{i}\right)}=\partial_{\mathbf{t}},
\end{aligned}
$$

where $A \sqcup B$ denotes the disjoint union of the sets $A$ and $B$. Notice that $\mathbf{t} \circledast_{i \in I}\left(\mathbf{t}_{i}, x_{i}\right)$ might not be compact.

Let us finish with an instance of a tree $\mathbf{t}$ such that $\mathcal{L}^{*}(\mathbf{t}) \neq \mathcal{L}(\mathbf{t})$.

Example 2.5. For every positive integer $n$, let us set $\mathbf{t}_{n}=[0,1 / n] \subset \mathbb{R}$, viewed as a rooted real tree when endowed with the usual distance on the real line and rooted at 0 . We consider the tree

$$
\mathbf{t}=\mathbf{t}_{1} \circledast_{n \geq 2}\left(\mathbf{t}_{n}, 1-\frac{1}{n^{2}}\right) .
$$

Then $\mathbf{t}$ is a compact height-regular tree and $1 \in \mathbf{t}_{1}$ is a leaf of $\mathbf{t}$ that does not belong to $\mathcal{L}^{*}(\mathbf{t})$.

2.2. The Gromov-Hausdorff topology. In order to define random real trees, we endow the set of (isometry classes of) rooted compact real trees with a metric, the 
so-called Gromov-Hausdorff metric, which hence defines a Borel $\sigma$-algebra on this set.

First, let us recall the definition of the Hausdorff distance between two compact subsets: let $A, B$ be two compact subsets of a metric space $\left(X, d_{X}\right)$. For every $\varepsilon>0$, we set:

$$
A^{\varepsilon}=\left\{x \in X, d_{X}(x, A) \leq \varepsilon\right\} .
$$

Then, the Hausdorff distance between $A$ and $B$ is defined by:

$$
d_{X, \text { Haus }}(A, B)=\inf \left\{\varepsilon>0, B \subset A^{\varepsilon} \text { and } A \subset B^{\varepsilon}\right\} .
$$

Now, let $\left(\mathbf{t}, d_{\mathbf{t}}, \partial_{\mathbf{t}}\right),\left(\mathbf{t}^{\prime}, d_{\mathbf{t}^{\prime}}, \partial_{\mathbf{t}^{\prime}}\right)$ be two compact rooted real trees. We define the pointed Gromov-Hausdorff distance between them, see Gromov (1999); Evans et al. (2006), by:

$$
d_{G H}\left(\mathbf{t}, \mathbf{t}^{\prime}\right)=\inf \left\{d_{Z, \text { Haus }}\left(\varphi(\mathbf{t}), \varphi^{\prime}(\mathbf{t})\right) \vee d_{Z}\left(\varphi\left(\partial_{\mathbf{t}}\right), \varphi^{\prime}\left(\partial_{\mathbf{t}^{\prime}}\right)\right)\right\},
$$

where the infimum is taken over all metric spaces $\left(Z, d_{Z}\right)$ and all isometric embed$\operatorname{dings} \varphi: \mathbf{t} \longrightarrow Z$ and $\varphi^{\prime}: \mathbf{t}^{\prime} \longrightarrow Z$.

Notice that $d_{G H}$ is only a pseudo-metric. We say that two rooted real trees $\mathbf{t}$ and $\mathbf{t}^{\prime}$ are equivalent (and we note $\mathbf{t} \sim \mathbf{t}^{\prime}$ ) if there exists a root-preserving isometry that maps $\mathbf{t}$ onto $\mathbf{t}^{\prime}$, that is $d_{G H}\left(\mathbf{t}, \mathbf{t}^{\prime}\right)=0$. This clearly defines an equivalence relation. We denote by $\mathbb{T}$ the set of equivalence classes of compact rooted real trees. The Gromov-Hausdorff distance $d_{G H}$ hence induces a metric on $\mathbb{T}$ (that is still denoted by $\left.d_{G H}\right)$. Moreover, the metric space $\left(\mathbb{T}, d_{G H}\right)$ is complete and separable, see Evans et al. (2006). If $\mathbf{t}, \mathbf{t}^{\prime}$ are two-compact rooted real trees such that $\mathbf{t} \sim \mathbf{t}^{\prime}$, then, for every $\varepsilon>0$, we have $r_{\varepsilon}(\mathbf{t}) \sim r_{\varepsilon}\left(\mathbf{t}^{\prime}\right)$. Thus, the erasure function $r_{\varepsilon}$ is well-defined on $\mathbb{T}$. It is easy to check that the functions $r_{\varepsilon}$ for $\varepsilon>0$ are continuous (and in fact Lipschitz).

Notice that if $\mathbf{t}$ is a compact height-regular real tree, so are all the trees equivalent to $\mathbf{t}$. Let $\mathbb{T}_{0} \subset \mathbb{T}$ denote the set of equivalence classes of compact binary heightregular real trees. The next lemma ensures that $\mathbb{T}_{0}$ is a Borel subset of $\mathbb{T}$.

Lemma 2.6. We have that $\mathbb{T}_{0}$ is a dense Borel subset of $\mathbb{T}$.

Proof: Let $\mathbb{T}^{f}$ (resp. $\mathbb{T}_{0}^{f}$ ) be the subset of trees of $\mathbb{T}$ (resp. $\mathbb{T}_{0}$ ) with finitely many leaves. Let $\varepsilon>0$. By Lemma 2.2, we have $r_{\varepsilon}(\mathbb{T}) \subset \mathbb{T}^{f}$. Conversely, for every $\mathbf{t} \in \mathbb{T}^{f}$, we define

$$
\tilde{\mathbf{t}}=\mathbf{t} \circledast_{x \in \mathcal{L}(\mathbf{t})}([0, \varepsilon], x)
$$

where the segment $[0, \varepsilon]$ is viewed as a rooted real tree when endowed with the usual distance on the real line and with root 0 . Then we have $r_{\varepsilon}(\tilde{\mathbf{t}})=\mathbf{t}$ and hence $r_{\varepsilon}(\mathbb{T})=\mathbb{T}^{f}$. The same arguments also apply to obtain $\mathbb{T}_{0}^{f}=r_{\varepsilon}\left(\mathbb{T}_{0}\right)$.

Notice that for every $n \geq 1$, the subset $\mathbb{T}^{n}$ of trees with less than $n$ leaves is a closed subset of $\mathbb{T}$ and that the subset of binary height-regular trees with exactly $n$ leaves is an open set (for the induced topology) of $\mathbb{T}^{n}$. This implies that $\mathbb{T}^{f}$ and $\mathbb{T}_{0}^{f}$ are Borel sets. We consider $r_{\varepsilon}$ as a function defined on $\mathbb{T}$. By definition, we have that $\mathbf{t} \in \mathbb{T}_{0}$ if and only if $r_{\varepsilon}(\mathbf{t}) \in \mathbb{T}_{0}$ for all $\varepsilon>0$, that is $\mathbb{T}_{0}=\bigcap_{\varepsilon>0} r_{\varepsilon}^{-1}\left(\mathbb{T}_{0}^{f}\right)$. Then use that $r_{\varepsilon}$ is continuous for all $\varepsilon>0$ to get that $\mathbb{T}_{0}$ is a measurable subset of $\mathbb{T}$.

Using Definition 2.3, it is easy to prove that $\mathbb{T}_{0}^{f}$ is dense in $\mathbb{T}^{f}$. Since

$$
d_{G H}\left(\mathbf{t}, r_{\varepsilon}(\mathbf{t})\right) \leq \varepsilon
$$


for $\mathbf{t} \in \mathbb{T}$ and $\varepsilon>0$, we deduce that $\mathbb{T}^{f}$ is dense in $\mathbb{T}$. This implies that $\mathbb{T}_{0}^{f}$, and thus $\mathbb{T}_{0}$, is dense in $\mathbb{T}$.

2.3. Brownian motion, local times and excursion theory. Let $\theta \geq 0, \beta>0$ and $B^{(\theta)}=\left(B_{t}^{(\theta)}, t \geq 0\right)$ be a Brownian motion with drift $-2 \theta$ and scale $\sqrt{2 / \beta}$ i.e. for $t \geq 0$

$$
B_{t}^{(\theta)}=\sqrt{2 / \beta} B_{t}-2 \theta t,
$$

where $B$ is a standard real Brownian motion.

For every $a \in \mathbb{R}$ and every $s \geq 0$, we define the local time $\Lambda_{s}^{a}$ of $B^{(\theta)}$ at time $s$ and level $a$ by approximation:

$$
\Lambda_{s}^{a}=\lim _{\varepsilon \rightarrow 0} \frac{1}{\varepsilon} \int_{0}^{s} \mathbf{1}_{\left\{a \leq B_{u}^{(\theta)} \leq a+\varepsilon\right\}} d u
$$

Moreover, there exists a continuous version of the bi-variate process $\left(\Lambda_{s}^{a}, s \geq 0, a \in\right.$ $\mathbb{R}$ ) and we always work with this continuous version. The local time process may also be characterized by the occupation time formula: for every nonnegative measurable function $\varphi$, we have a.s.

$$
\int_{0}^{t} \varphi\left(B_{s}^{(\theta)}\right) d s=\int_{\mathbb{R}} \varphi(x) \Lambda_{t}^{x} d x .
$$

For every $a \in \mathbb{R}$, the function $s \longmapsto \Lambda_{s}^{a}$ is a.s. non-decreasing and we denote by $d \Lambda_{s}^{a}$ the associated Stieljes measure.

Let $\mathcal{E}$ be the set of positive excursions i.e. the set of continuous functions $g$ : $[0,+\infty) \longrightarrow[0,+\infty)$ with compact support and such that $g(0)=0$. For $g \in \mathcal{E}$, we set $\sigma(g)=\sup \{x, g(x)>0\}$ the duration or the length of the excursion $g$.

In order to define the Itô measure of positive excursions of the Brownian motion with drift, we consider the Brownian motion $B^{(\theta)}$ reflected at 0 , i.e. the process $\mathrm{H}=\left(\mathrm{H}_{t}, t \geq 0\right)$ defined by:

$$
\mathrm{H}_{t}:=B_{t}^{(\theta)}-I_{t}^{(\theta)}
$$

where $I_{t}^{(\theta)}$ is the infimum process of $B^{(\theta)}$ :

$$
I_{t}^{(\theta)}=\inf _{u \in[0, t]} B_{u}^{(\theta)} .
$$

The process $\left(-I_{t}^{(\theta)}, t \geq 0\right)$ is a continuous non-decreasing process, and the set of increase points coincides with the set

$$
\mathcal{Z}=\left\{t \geq 0, \mathrm{H}_{t}=0\right\}
$$

of zeros of $\mathrm{H}$. We introduce the right-continuous inverse of the infimum process,

$$
\tau_{u}=\inf \left\{t \geq 0, I_{t}^{(\theta)}<-u\right\}
$$

and we define the set $D$ of discontinuities of $\tau$. For every $u \in D$, we define the excursion $e_{u}=\left(e_{u}(t), t \geq 0\right) \in \mathcal{E}$ of $\mathrm{H}$ away from 0 associated with the interval $\left(\tau_{u-}, \tau_{u}\right)$ by

$$
e_{u}(t)= \begin{cases}\mathrm{H}_{\tau_{u-}+t} & \text { if } 0 \leq t \leq \tau_{u}-\tau_{u-}, \\ 0 & \text { if } t>\tau_{u}-\tau_{u-} .\end{cases}
$$

Then the point measure $\sum_{u \in D} \delta_{\left(u, e_{u}\right)}(d s d e)$ is a Poisson point measure on $\mathbb{R}_{+} \times \mathcal{E}$ with intensity $d s \otimes n^{(\theta)}(d e)$ where $n^{(\theta)}(d e)$ is a $\sigma$-finite measure on $\mathcal{E}$ called Itô's measure of positive excursions of $B^{(\theta)}$. 
With that definition, we have for $\lambda \geq 0$ :

$$
n^{(\theta)}\left[1-\mathrm{e}^{-\lambda \sigma}\right]=\psi_{\theta}^{-1}(\lambda),
$$

where

$$
\psi_{\theta}(\lambda)=\beta \lambda^{2}+2 \beta \theta \lambda .
$$

Let $\zeta=\zeta(e)=\max _{s \in[0, \sigma]}\left(e_{s}\right)$ be the maximum of the excursion. We set $c_{\theta}(h)=$ $n^{(\theta)}[\zeta \geq h]$ for $h>0$, and we recall, see Section 7 in Chen and Delmas (2012) for the case $\theta>0$, that:

$$
c_{\theta}(h)= \begin{cases}(\beta h)^{-1} & \text { if } \theta=0 \\ 2 \theta\left(\mathrm{e}^{2 \beta \theta h}-1\right)^{-1} & \text { if } \theta>0\end{cases}
$$

2.4. Coding a compact real tree by a function and the Brownian CRT. Let $g \in \mathcal{E}$, and assume that $\sigma(g)>0$, that is $g$ is not identically zero. For every $s, t \geq 0$, we set

$$
m_{g}(s, t)=\inf _{r \in[s \wedge t, s \vee t]} g(r)
$$

and

$$
d_{g}(s, t)=g(s)+g(t)-2 m_{g}(s, t) .
$$

It is easy to check that $d_{g}$ is a pseudo-metric on $[0,+\infty)$. We then say that $s$ and $t$ are equivalent iff $d_{g}(s, t)=0$ and we set $T_{g}$ the associated quotient space. We keep the notation $d_{g}$ for the induced distance on $T_{g}$. Then the metric space $\left(T_{g}, d_{g}\right)$ is a compact real-tree, see Duquesne and Le Gall (2005). We denote by $p_{g}$ the canonical projection from $[0,+\infty)$ to $T_{g}$. We will view $\left(T_{g}, d_{g}\right)$ as a rooted real tree with root $\partial=p_{g}(0)$. We will call $\left(T_{g}, d_{g}\right)$ the real tree coded by $g$, and conversely that $g$ is a contour function of the tree $T_{g}$. We denote by $F$ the application that associates with a function $g \in \mathcal{E}$ the equivalence class of the tree $T_{g}$.

Conversely every rooted compact real tree $(T, d)$ can be coded by a continuous function $g$ (up to a root-preserving isometry), see Duquesne (2006).

For $\theta \geq 0$, we define the Brownian CRT, $\tau=F(e)$, as the (equivalence class of the) tree coded by the positive excursion $e$ under $n^{(\theta)}$. And we define the measure $\mathbb{N}^{(\theta)}$ on $\mathbb{T}$ as the "distribution" of $\tau$, that is the push-forward of the measure $n^{(\theta)}$ by the application $F$. Notice that $H(\tau)=\zeta(e)$.

Remark 2.7. If we translate the former construction into the framework of Duquesne and Le Gall (2002), then $\mathrm{H}$ is the height process which codes the Brownian CRT with branching mechanism $\psi_{\theta}$ and it is obtained from the underlying Lévy process $X=\left(X_{t}, t \geq 0\right)$ with $X_{t}=\sqrt{2 \beta} B_{t}-2 \beta \theta t$.

As for $B^{(\theta)}$, we can consider under $n^{(\theta)}(d e)$ the local time $\Lambda_{s}^{a}$ of $e$ at time $s$ and level $a$. Then we define the local time measure of $\tau$ at level $a \geq 0$, denoted by $\ell_{a}(d x)$, as the push-forward of the measure $d \Lambda_{s}^{a}$ by the map $F$, see Theorem 4.2 in Duquesne and Le Gall (2005). We shall define $\ell_{a}$ for $a \in \mathbb{R}$ by setting $\ell_{a}=0$ for $a \in \mathbb{R} \backslash[0, H(\tau)]$. 
2.5. Forests. A forest $\mathbf{f}$ is a family $\left(\left(h_{i}, \mathbf{t}_{i}\right), i \in I\right)$ of points of $\mathbb{R} \times \mathbb{T}$, where $I$ is an at most countable index set. Using an immediate extension of the grafting procedure, for an interval $\mathfrak{I} \subset \mathbb{R}$, we define the real tree $\mathbf{f}_{\mathfrak{I}}=\mathfrak{I} \circledast_{i \in I, h_{i} \in \mathfrak{I}}\left(\mathbf{t}_{i}, h_{i}\right)$. For $\mathfrak{I}=\mathbb{R}, \mathbf{f}_{\mathbb{R}}$ is an infinite spine (the real line) on which we graft the compact trees $\mathbf{t}_{i}$ at the points $h_{i}$ respectively. We shall identify the forest $\mathbf{f}$ with $\mathbf{f}_{\mathbb{R}}$ when the $\left(h_{i}, i \in I\right)$ are pairwise distinct.

Let us denote, for $i \in I$, by $d_{i}$ the distance of the tree $\mathbf{t}_{i}$ and by $\mathbf{t}_{i}^{\circ}=\mathbf{t}_{i} \backslash\left\{\partial_{\mathbf{t}_{i}}\right\}$ the tree $\mathbf{t}_{i}$ without its root. The distance on $\mathbf{f}_{\mathfrak{I}}$ is then defined, for $x, y \in \mathbf{f}_{\mathfrak{I}}$, by:

$$
d_{\mathbf{f}}(x, y)= \begin{cases}d_{i}(x, y) & \text { if } x, y \in \mathbf{t}_{i}^{\circ}, \\ h_{\mathbf{t}_{i}}(x)+\left|h_{i}-h_{j}\right|+h_{\mathbf{t}_{j}}(y) & \text { if } x \in \mathbf{t}_{i}^{\circ}, y \in \mathbf{t}_{j}^{\circ} \text { with } i \neq j, \\ \left|x-h_{j}\right|+h_{\mathbf{t}_{j}}(y) & \text { if } x \notin \bigcup_{i \in I} \mathbf{t}_{i}^{\circ}, y \in \mathbf{t}_{j}^{\circ} \\ |x-y| & \text { if } x, y \notin \bigcup_{i \in I} \mathbf{t}_{i}^{\circ} .\end{cases}
$$

The next lemma essentially states that $\mathbf{f}_{\mathbb{R}}$ is locally compact. See Abraham et al. (2013) and the references therein for the Gromov-Hausdorff topology on the set of locally compact trees.

Lemma 2.8. Let $\mathfrak{I} \subset \mathbb{R}$ be a closed interval. If for every $a, b \in \mathfrak{I}$, such that $a<b$, and every $\varepsilon>0$, the set $\left\{i \in I, h_{i} \in[a, b], H\left(\mathbf{t}_{i}\right)>\varepsilon\right\}$ is finite, then the tree $\mathbf{f}_{\mathfrak{I}}$ is a complete locally compact real tree.

Proof: Let $\left(x_{n}, n \geq 0\right)$ be a bounded sequence of $\mathbf{f}_{\mathfrak{I}}$. If there exists a sub-sequence $\left(x_{n_{k}}, k \geq 0\right.$ ) which belongs to $\mathfrak{I}$ (resp. to $\mathbf{t}_{i}^{\circ}$ for some $i \in I$ ), then as $\mathfrak{I}$ is a closed interval (resp. $\mathbf{t}_{i}^{\circ} \cup\left\{h_{i}\right\}$ is compact), this sub-sequence admits at least one accumulation point.

If this is not the case, without loss of generality, we can suppose that $x_{n} \in \mathbf{t}_{i_{n}}^{\circ}$ with pairwise distinct indices $i_{n}$. Notice that the sequence $\left(h_{i_{n}}, n \geq 0\right)$ of elements of $\mathfrak{I}$ is bounded, since $d_{\mathbf{f}}\left(h_{i_{0}}, h_{i_{n}}\right) \leq d_{\mathbf{f}}\left(x_{0}, x_{n}\right)$. Therefore, as $\mathfrak{I}$ is a closed interval, there exists a converging sub-sequence $\left(h_{i_{n_{k}}}, k \geq 0\right)$. Let us denote by $h \in \mathfrak{I}$ its limit. Moreover, using the assumption that $\left\{i \in I, h_{i} \in[a, b], H\left(\mathbf{t}_{i}\right)>\varepsilon\right\}$ is finite for all $a<b$, we have $\lim _{n \rightarrow+\infty} d_{\mathbf{f}}\left(x_{n}, h_{i_{n}}\right)=0$. Therefore, the sub-sequence $\left(x_{n_{k}}, k \geq 0\right)$ converges to $h$.

In conclusion, we get that every bounded sequence of $\mathbf{f}_{\mathfrak{I}}$ admits at least one accumulation point. This implies that $\mathbf{f}_{\mathfrak{I}}$ is complete and locally compact.

We extend the notion of height of a vertex and of the subtree above a vertex for a forest: for $x \in \mathbf{f}_{\mathbb{R}}$, either there exists a unique $i \in I$ such that $x \in \mathbf{t}_{i}$ and we set $h_{\mathbf{f}}(x)=h_{i}+h_{\mathbf{t}_{i}(x)}$ and $\mathbf{t}_{x}$ the subtree above $x$ in $\mathbf{t}_{i}$, or $x \in \mathbb{R}$ and we set $h_{\mathbf{f}}(x)=x$ and $\mathbf{t}_{x}=\{x\}$.

\section{The reversed tree}

3.1. Backbones. For a compact rooted real tree $\mathbf{t}$, we define an increasing family of backbones $\left(B_{n}(\mathbf{t})\right)_{n \in \mathbb{N}}$. We denote by $S_{0}(\mathbf{t})=\left\{x \in \mathbf{t}, h_{\mathbf{t}}(x)=H(\mathbf{t})\right\}$ the set of leaves with maximal height and we define the initial backbone as the set of ancestors of $S_{0}(\mathbf{t})$ :

$$
B_{0}(\mathbf{t})=\bigcup_{x \in S_{0}(\mathbf{t})} \llbracket \partial, x \rrbracket .
$$

Notice that if the tree $\mathbf{t}$ is height-regular, then $S_{0}(\mathbf{t})=\left\{\partial^{*}\right\}$ and $B_{0}(\mathbf{t})=\llbracket \partial, \partial^{*} \rrbracket$ is just the spine from the root of the tree to its top. 
Let $\left(\tilde{\mathbf{t}}^{i}, i \in I_{0}\right)$ be the connected components of $\mathbf{t} \backslash B_{0}(\mathbf{t})$. If $\mathbf{t}^{i}$ denotes the closure of $\tilde{\mathbf{t}}^{i}$, we have $\mathbf{t}^{i}=\tilde{\mathbf{t}}^{i} \cup\left\{x_{i}\right\}$ for a unique $x_{i} \in B_{0}(\mathbf{t})$ which can be viewed as the root of $\mathbf{t}^{i}$. Then, we define the family of backbones recursively: for $n \geq 1$, we set

$$
B_{n}(\mathbf{t})=B_{0}(\mathbf{t}) \circledast \circledast_{i \in I_{0}}\left(B_{n-1}\left(\mathbf{t}^{i}\right), x_{i}\right) .
$$

Remark 3.1. We can also use the alternative recursive definition

$$
B_{n}(\mathbf{t})=B_{n-1}(\mathbf{t}) \circledast_{i \in I_{n-1}}\left(B_{0}\left(\hat{\mathbf{t}}^{i} \cup\left\{y_{i}\right\}\right), y_{i}\right),
$$

where the family $\left(\hat{\mathbf{t}}^{i}, i \in I_{n-1}\right)$ is the connected components of $\mathbf{t} \backslash B_{n-1}(\mathbf{t})$ and $y_{i}$ is the unique vertex of $\mathbf{t}$ such that $\hat{\mathbf{t}}^{i} \cup\left\{y_{i}\right\}$ is closed (and $y_{i}$ is then considered as the root of this tree).

Remark 3.2. It is easy to check that, if $\mathbf{t} \sim \mathbf{t}^{\prime}$ then, for every $n \in \mathbb{N}, B_{n}(\mathbf{t}) \sim B_{n}\left(\mathbf{t}^{\prime}\right)$. So the function $B_{n}$ is well defined on $\mathbb{T}$.

It is easy to check that for $\mathbf{t}$ a compact rooted real tree, $\varepsilon>0$ :

$$
r_{\varepsilon} \circ B_{n}(\mathbf{t})=B_{n} \circ r_{\varepsilon}(\mathbf{t}) .
$$

By Lemma 2.2, we deduce that for every $\mathbf{t} \in \mathbb{T}$ and $\varepsilon>0$, there exists an integer $N$ (that depends on $\mathbf{t}$ and $\varepsilon$ ) such that

$$
r_{\varepsilon}(\mathbf{t})=\bigcup_{n=0}^{N} B_{n}\left(r_{\varepsilon}(\mathbf{t})\right)=B_{N} \circ r_{\varepsilon}(\mathbf{t}) .
$$

Lemma 3.3. Let $\mathbf{t}$ be a compact rooted real tree not reduced to the root.

(i) We have cl $\left(\bigcup_{n \in \mathbb{N}} B_{n}(\mathbf{t})\right)=\mathbf{t}$.

(ii) If $\mathbf{t}$ is height-regular and binary, then we have $\bigcup_{n \in \mathbb{N}} \mathcal{L}\left(B_{n}(\mathbf{t})\right)=\mathcal{L}^{*}(\mathbf{t})$.

(iii) We have $\lim _{n \rightarrow \infty} d_{G H}\left(B_{n}(\mathbf{t}), \mathbf{t}\right)=0$.

Proof: We prove (i). Let $\mathbf{t}$ be a compact rooted real tree not reduced to the root. Let $x \in \operatorname{sk}(\mathbf{t})$ and set $\varepsilon=H\left(\mathbf{t}_{x}\right)>0$. By definition $x \in r_{\varepsilon}(\mathbf{t})$, and using (3.3) as well as the inclusion $B_{n}\left(r_{\varepsilon}(\mathbf{t})\right) \subset B_{n}(\mathbf{t})$, we get $x \in \bigcup_{n \in \mathbb{N}} B_{n}(\mathbf{t})$, which proves that $\operatorname{sk}(\mathbf{t}) \subset \bigcup_{n \in \mathbb{N}} B_{n}(\mathbf{t})$. Then the first point follows from the fact that $\mathrm{cl}(\operatorname{sk}(\mathbf{t}))=\mathbf{t}$.

We prove (ii). Let us suppose that $\mathbf{t}$ is height-regular and binary, and let $x \in$ $\mathcal{L}\left(B_{n}(\mathbf{t})\right)$ for some $n \in \mathbb{N}$. Then, by definition of $B_{n}(\mathbf{t}), x$ is the top of a subtree of the form $\mathbf{t}_{y}$, with $y \prec x$ and, as $\mathbf{t}$ is height-regular, it therefore belongs to $\mathcal{L}^{*}(\mathbf{t})$. Conversely, let $x \in \mathcal{L}^{*}(\mathbf{t})$. Then there exists $y \in \operatorname{sk}(\mathbf{t})$ such that $y^{*}=x$. Let us set $\varepsilon=d(y, x)>0$. Then $y \in r_{\varepsilon}(\mathbf{t})$ and, by (3.3), $y \in B_{n}(\mathbf{t})$ for some $n \in \mathbb{N}$. And by definition, we have $x=y^{*} \in \mathcal{L}\left(B_{n}(\mathbf{t})\right)$ for the same $n$.

We prove (iii). Using notation from Remark 3.1, we deduce from (3.1) that $d_{\mathbf{t} \text {,Haus }}\left(B_{n}(\mathbf{t}), \mathbf{t}\right) \leq \sup _{i \in I_{n}} H\left(\hat{\mathbf{t}}^{i} \cup\left\{y_{i}\right\}\right)$. Then use that $\mathbf{t}$ is compact to get:

$$
\lim _{n \rightarrow \infty} \sup _{i \in I_{n}} H\left(\hat{\mathbf{t}}^{i} \cup\left\{y_{i}\right\}\right)=0 .
$$

This gives the result.

3.2. Reversed tree. The reversal of a tree will be defined only on $\mathbb{T}_{0}$. Let $\mathbf{t}$ be a rooted compact height-regular binary trees. As already noticed, since $\mathbf{t}$ is height regular, we have $S_{0}(\mathbf{t})=\left\{\partial^{*}\right\}$ and $B_{0}(\mathbf{t})=\llbracket \partial, \partial^{*} \rrbracket$. Similarly, using the notations of Section 3.1, for every $i \in I_{0}$, as $\mathbf{t}^{i}$ is also height-regular, we have $B_{0}\left(\mathbf{t}^{i}\right)=\llbracket x_{i}, x_{i}^{*} \rrbracket$. 


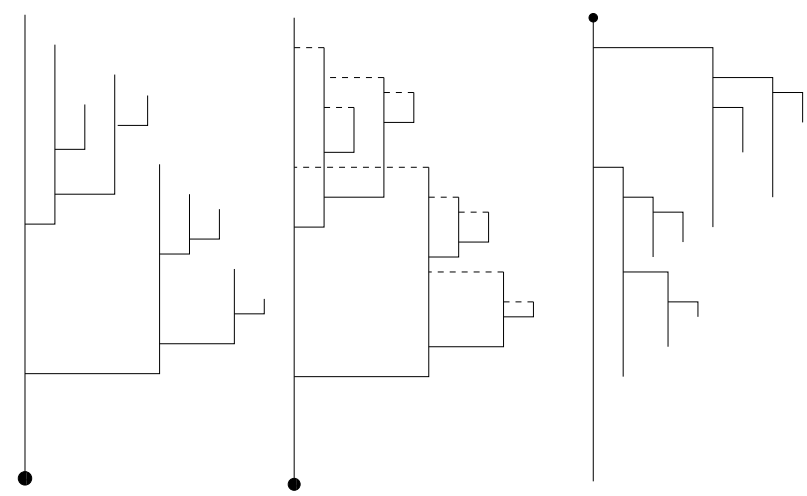

Figure 3.2. A backbone $B_{3}(\mathbf{t})$ on the left and its reversed tree $\mathcal{R}_{3}(\mathbf{t})$ on the right. The root of each tree is represented by a bullet.

For every $i \in I_{0}$, we set $y_{i}^{\prime}$ the unique point of $B_{0}(\mathbf{t})$ which is at the same height as $x_{i}^{*}$ :

$$
y_{i}^{\prime} \in \llbracket \partial, \partial^{*} \rrbracket, h_{\mathbf{t}}\left(y_{i}^{\prime}\right)=h_{\mathbf{t}}\left(x_{i}^{*}\right) .
$$

We then define recursively the reversed backbones as follows. We set, for $n \geq 0$,

$$
\mathcal{R}_{0}(\mathbf{t})=\left(\llbracket \partial^{*}, \partial \rrbracket, d, \partial^{*}\right) .
$$

(notice that the root of $\mathcal{R}_{0}(\mathbf{t})$ is $\partial^{*}$ ) and for $n \geq 1$,

$$
\mathcal{R}_{n}(\mathbf{t})=\mathcal{R}_{0}(\mathbf{t}) \circledast_{i \in I_{0}}\left(\mathcal{R}_{n-1}\left(\mathbf{t}^{i}\right), y_{i}^{\prime}\right) .
$$

The reversal procedure is illustrated on Figure 3.2, the dashed lines show where the trees are grafted on the reversed tree. Notice that, for aesthetic purpose, inside a sub-tree, the branches are drawn from left to right in decreasing order of their height.

Intuitively, the leaves of $\mathcal{R}_{n}(\mathbf{t})$ correspond to branching points of $B_{n}(\mathbf{t})$ (or to its root) and conversely. Therefore, it is easy to check that $\mathcal{R}_{n}(\mathbf{t}) \in \mathbb{T}_{0}$ for every $n \in \mathbb{N}$.

Lemma 3.4. Let $\mathbf{t}$ be a compact rooted height-regular binary tree. We have:

$$
\mathcal{R}_{n} \circ B_{n}(\mathbf{t})=\mathcal{R}_{n}(\mathbf{t}), \quad \mathcal{R}_{n} \circ \mathcal{R}_{n}(\mathbf{t})=B_{n}(\mathbf{t}) \quad \text { and } \quad \mathcal{R}_{n} \circ r_{\varepsilon}(\mathbf{t})=r_{\varepsilon} \circ \mathcal{R}_{n}(\mathbf{t})
$$

Proof: The first two equalities are obvious and the last one is also obvious if $\mathbf{t}$ has a finite number of leaves. We just check that the last equality holds for general $\mathbf{t} \in \mathbb{T}_{0}$. We have:

$$
\mathcal{R}_{n} \circ r_{\varepsilon}(\mathbf{t})=\mathcal{R}_{n} \circ B_{n} \circ r_{\varepsilon}(\mathbf{t})=\mathcal{R}_{n} \circ r_{\varepsilon} \circ B_{n}(\mathbf{t})=r_{\varepsilon} \circ \mathcal{R}_{n} \circ B_{n}(\mathbf{t})=r_{\varepsilon} \circ \mathcal{R}_{n}(\mathbf{t}),
$$

where we use the first equality of (3.5) for the first equality, (3.2) for the second, the last equality of (3.5) which holds for $B_{n}(\mathbf{t})$ as it is height-regular and has a finite number of leaves for the third and (3.5) for the last.

The sequence of trees $\left(\mathcal{R}_{n}(\mathbf{t}), n \geq 0\right)$ is non-decreasing. We endow $\bigcup_{n>0} \mathcal{R}_{n}(\mathbf{t})$ with the natural distance denoted by $d^{\mathcal{R}}$ and we define the reversed tree $\mathcal{R}(\mathbf{t})$ as the completion of $\bigcup_{n \geq 0} \mathcal{R}_{n}(\mathbf{t})$ with respect to the distance $d^{\mathcal{R}}$. 
If $\mathbf{t}$ and $\mathbf{t}^{\prime}$ are equivalent, that is $\mathbf{t} \sim \mathbf{t}^{\prime}$, we get that $\mathcal{R}_{n}(\mathbf{t}) \sim \mathcal{R}_{n}\left(\mathbf{t}^{\prime}\right)$ for all $n \in \mathbb{N}$ and $\mathcal{R}(\mathbf{t}) \sim \mathcal{R}\left(\mathbf{t}^{\prime}\right)$ as well. Thus the functions $\left(\mathcal{R}_{n}, n \in \mathbb{N}\right)$ and $\mathcal{R}$ are well defined on $\mathbb{T}_{0}$. We give some properties of the map $\mathcal{R}$.

Corollary 3.5. We have that $\mathcal{R}=\lim _{n \rightarrow \infty} \mathcal{R}_{n}$ on $\left(\mathbb{T}_{0}, d_{G H}\right)$ for the simple convergence. The map $\mathcal{R}$ is a one-to-one measurable involution (that is $\mathcal{R} \circ \mathcal{R}(\mathbf{t})=\mathbf{t}$ ) defined on $\mathbb{T}_{0}$.

Proof: Let $\mathbf{t}$ be a compact rooted height-regular binary tree. Using notation from Remark 3.1, we deduce from (3.1) that $d_{\mathbf{t}, \text { Haus }}\left(B_{n}(\mathbf{t}), B_{m}(\mathbf{t})\right) \leq \sup _{i \in I_{n}} H\left(\hat{\mathbf{t}}^{i} \cup\left\{y_{i}\right\}\right)$ for $m \geq n$. This in turns implies that $d_{\mathcal{R}(\mathbf{t}), \text { Haus }}\left(\mathcal{R}_{n}(\mathbf{t}), \mathcal{R}_{m}(\mathbf{t})\right) \leq \sup _{i \in I_{n}} H\left(\hat{\mathbf{t}}^{i} \cup\right.$ $\left.\left\{y_{i}\right\}\right)$ for $m \geq n$. Use (3.4) to deduce that $\left(\mathcal{R}_{n}(\mathbf{t}), n \in \mathbb{N}\right)$ is a Cauchy sequence in $\mathbb{T}_{0}$. Thanks to Lemma 2.7 in Evans et al. (2006), we get that

$$
\lim _{n \rightarrow \infty} d_{G H}\left(\mathcal{R}_{n}(\mathbf{t}), \mathcal{R}(\mathbf{t})\right)=0,
$$

which implies that $\mathcal{R}=\lim _{n \rightarrow \infty} \mathcal{R}_{n}$ on $\left(\mathbb{T}_{0}, d_{G H}\right)$ for the simple convergence.

By construction, for all $\mathbf{t} \in \mathbb{T}_{0}^{f}$, the set of compact height-regular binary trees with a finite number of leaves, there exists $n \in \mathbb{N}$ such that $\mathcal{R}_{n}(\mathbf{t})=\mathcal{R}(\mathbf{t})$ and $B_{n}(\mathbf{t})=\mathbf{t}$. Thus, thanks to the second equality in (3.5), we deduce that $\mathcal{R}$ is an involution on $\mathbb{T}_{0}^{f}$. Using that $r_{\varepsilon}\left(\mathbb{T}_{0}\right) \subset \mathbb{T}_{0}^{f}$, we deduce from the third equality in (3.5) and the continuity of $r_{\varepsilon}$ that $r_{\varepsilon} \circ \mathcal{R}=\mathcal{R} \circ r_{\varepsilon}$ on $\mathbb{T}_{0}$ and thus:

$$
r_{\varepsilon} \circ \mathcal{R} \circ \mathcal{R}=\mathcal{R} \circ \mathcal{R} \circ r_{\varepsilon}=r_{\varepsilon} .
$$

As $\lim _{\varepsilon \rightarrow 0} r_{\varepsilon}(\mathbf{t})=\mathbf{t}$, we deduce from the above equalities, by letting $\varepsilon$ goes down to 0 , that $\mathcal{R} \circ \mathcal{R}(\mathbf{t})=\mathbf{t}$ for all $\mathbf{t} \in \mathbb{T}_{0}$.

It is easy to see that $\mathcal{R}$ is continuous when restricted to $r_{\varepsilon}\left(\mathbb{T}_{0}\right)$. This gives that $\mathcal{R} \circ r_{\varepsilon}$ is measurable. Thus as $r_{\varepsilon} \circ \mathcal{R}=\mathcal{R} \circ r_{\varepsilon}$, we get that $r_{\varepsilon} \circ \mathcal{R}$ is measurable. Then, use that $\mathcal{R}=\lim _{\varepsilon \rightarrow 0} r_{\varepsilon} \circ \mathcal{R}$ to deduce that $\mathcal{R}$ is measurable on $\mathbb{T}_{0}$.

Remark 3.6. There is no natural extension of $\mathcal{R}$ to $\mathbb{T}$ (in particular because $\mathcal{R}$ is not uniformly continuous of $\mathbb{T}_{0}$ ).

\subsection{Reversed CRT. We first check that the Brownian CRT is height-regular.}

Lemma 3.7. Let $\theta \geq 0$. Let $\tau$ be a Brownian CRT under the excursion measure $\mathbb{N}^{(\theta)}$. Then, we have that $\mathbb{N}^{(\theta)}$-a.e., $\tau \in \mathbb{T}_{0}$.

Proof: Let $h>0$. Following Neveu and Pitman (1989a,b), we say that a process $X$ admits a $h$-minimum (resp. a $h$-maximum) at time $t$ if there exist $s<t$ and $u>t$ such that $X_{s}=X_{u}=X_{t}+h\left(\right.$ resp. $\left.X_{s}=X_{u}=X_{t}-h\right)$ and $X_{r} \geq X_{t}$ (resp. $\left.X_{r} \leq X_{t}\right)$ for every $r \in[s, u]$.

Then, if we denote by $e$ an excursion under $n^{(\theta)}$ and $\tau$ the associated real tree, for a.e. $h$ the branching points of $r_{h}(\tau)$ correspond to the $h$-minima of $e$ and each leaf of $r_{h}(\tau)$ is associated with an $h$-maxima of $e$. As $n^{(\theta)}$-a.e., two local extrema of the excursion $e$ have different levels, we get that $r_{h}(\tau)$ belongs a.e. to $\mathbb{T}_{0}$ for all $h>0$ that is, by definition of $\mathbb{T}_{0}, \mathbb{N}^{(\theta)}$-a.e., $\tau \in \mathbb{T}_{0}$.

Let $\tau$ be a Brownian CRT under the excursion measure $\mathbb{N}^{(\theta)}$, with $\theta \geq 0$. We keep the notations of Section 3.1: we set $B_{0}(\tau)=\llbracket \partial, \partial^{*} \rrbracket$ and set $\left(\tau_{i}, i \in I_{0}\right)$ the closures of the connected components of $\tau \backslash B_{0}(\tau)$ viewed as trees in $\mathbb{T}$ rooted respectively at point $x_{i} \in B_{0}(\tau)$ so that $\tau=B_{0}(\tau) \circledast_{i \in I_{0}}\left(\tau_{i}, x_{i}\right)$. 
Lemma 3.8. Let $\theta \geq 0$. Under $\mathbb{N}^{(\theta)}$, the point measure $\sum_{i \in I_{0}} \delta_{\left(h-u_{i}-H\left(\tau_{i}\right), \tau_{i}\right)}$ on $[0, h] \times \mathbb{T}$ is, conditionally given $\{H(\tau)=h\}$, a Poisson point measure with intensity

$$
2 \beta \mathbf{1}_{(0, h)}(u) d u \mathbb{N}^{(\theta)}[d \mathbf{t}, H(\mathbf{t}) \leq h-u] .
$$

Proof: By the Williams decomposition, see Abraham and Delmas (2009), the point measure $\sum_{i \in I_{0}} \delta_{\left(u_{i}, \tau_{i}\right)}$ is under $\mathbb{N}^{(\theta)}$, conditionally given $\{H(\tau)=h\}$, a Poisson point measure with intensity (3.6). Then, for every non-negative function $\varphi$ on $[0, h] \times \mathbb{T}$, we have

$$
\begin{aligned}
\mathbb{N}^{(\theta)}\left[\mathrm{e}^{-\sum_{i \in I_{0}} \varphi\left(h-u_{i}-H\left(\tau_{i}\right), \tau_{i}\right)} \mid H(\tau)=h\right] \\
=\exp \left(-\int_{0}^{h} 2 \beta d u \mathbb{N}^{(\theta)}\left[\left(1-\mathrm{e}^{-\varphi(h-u-H(\tau), \tau)}\right) \mathbf{1}_{\{H(\tau) \leq h-u\}}\right]\right) \\
=\exp \left(-2 \beta \mathbb{N}^{(\theta)}\left[\int_{0}^{h-H(\tau)} d u\left(1-\mathrm{e}^{-\varphi(h-u-H(\tau), \tau)}\right) \mathbf{1}_{\{H(\tau) \leq h\}}\right]\right) \\
=\exp \left(-2 \beta \mathbb{N}^{(\theta)}\left[\int_{0}^{h-H(\tau)} d v\left(1-\mathrm{e}^{-\varphi(v, \tau)}\right) \mathbf{1}_{\{H(\tau) \leq h\}}\right]\right) \\
=\exp \left(-\int_{0}^{h} 2 \beta d v \mathbb{N}^{(\theta)}\left[\left(1-\mathrm{e}^{-\varphi(v, \tau)}\right) \mathbf{1}_{\{H(\tau) \leq h-v\}}\right]\right),
\end{aligned}
$$

where we performed the change of variables $v=h-u-H(\tau)$ for the third equality. The lemma follows.

Theorem 3.9. Let $\theta \geq 0$. Let $\tau$ be a Brownian CRT under the excursion measure $\mathbb{N}^{(\theta)}$. Then, $\mathcal{R}(\tau)$ is distributed as $\tau$.

Proof: To prove the theorem, it suffices to prove, using Lemma 3.3, that for every $n \in \mathbb{N}, B_{n}(\tau)$ and $\mathcal{R}_{n}(\tau)$ are equally distributed, which we prove by induction.

First, as $\mathbb{N}^{(\theta)}$-a.e. $\tau \in \mathbb{T}_{0}$, we have $B_{0}(\tau)=\mathcal{R}_{0}(\tau)$ (viewed as equivalence classes). They have consequently the same distribution.

Suppose now that $B_{n-1}(\tau)$ and $\mathcal{R}_{n-1}(\tau)$ are equally distributed for some $n \geq 1$. Recall that

$$
B_{n}(\tau)=B_{0}(\tau) \circledast_{i \in I_{0}}\left(B_{n-1}\left(\tau_{i}\right), x_{i}\right) \quad \text { and } \quad \mathcal{R}_{n}(\tau)=\mathcal{R}_{0}(\tau) \circledast_{i \in I_{0}}\left(\mathcal{R}_{n-1}\left(\tau_{i}\right), y_{i}^{\prime}\right),
$$

where for every $i \in I_{0}, y_{i}^{\prime}$ is the unique point of $B_{0}(\tau)$ which has the same height as $x_{i}^{*}$ i.e. such that $h_{\tau}\left(y_{i}^{\prime}\right)=h_{\tau}\left(x_{i}\right)+H\left(\tau_{i}\right)$. Notice that, as a vertex of $\mathcal{R}_{0}(\tau), y_{i}^{\prime}$ has height $h_{\mathcal{R}_{0}(\tau)}\left(y_{i}^{\prime}\right)=H(\tau)-h_{\tau}\left(x_{i}\right)-H\left(\tau_{i}\right)$.

Thanks to Lemma 3.8, conditionally given $B_{0}(\tau)$, the two families

$$
\left(\left(h_{\tau}\left(x_{i}\right), \tau_{i}\right), i \in I_{0}\right) \quad \text { and } \quad\left(\left(h_{\mathcal{R}_{0}(\tau)}\left(y_{i}^{\prime}\right), \tau_{i}\right), i \in I_{0}\right)
$$

have the same distribution. By the induction assumption, the families

$$
\left(\left(h_{\tau}\left(x_{i}\right), B_{n-1}\left(\tau_{i}\right)\right), i \in I_{0}\right) \quad \text { and } \quad\left(\left(h_{\mathcal{R}_{0}(\tau)}\left(y_{i}^{\prime}\right), \mathcal{R}_{n-1}\left(\tau_{i}\right)\right), i \in I_{0}\right)
$$

have also the same distribution. This implies that, under $\mathbb{N}^{(\theta)}, B_{n}(\tau)$ and $\mathcal{R}_{n}(\tau)$ are equally distributed.

The reversal operation is natural on the Brownian CRT but it has no elementary representation for the underlying Brownian excursion. Informally, one can think of a Vervaat transform for every sub-excursion above every level. 
Recall the definition in Section 2.4 of the local time measure $\ell_{a}(d x)$ of a Brownian CRT $\tau$ at level $a$. We denote by $\ell_{a}(\tau)$ the total mass of this measure. We recover the time-reversal distribution invariance of the local time of the Brownian excursion.

Corollary 3.10. Let $\theta \geq 0$. $\mathbb{N}^{(\theta)}$-a.e., for every $a \geq 0, \ell_{a}(\tau)=\ell_{H(\tau)-a}(\mathcal{R}(\tau))$.

Proof: Let $a>0$. Using Theorem 4.2 of Duquesne and Le Gall (2005), we have that $\mathbb{N}^{(\theta)}$-a.e.:

$\ell_{a}(\tau)=\lim _{\varepsilon \rightarrow 0} \frac{1}{\varepsilon} \operatorname{Card}\left\{x \in r_{\varepsilon}(\tau), h_{\tau}(x)=a-\varepsilon\right\}=\lim _{\varepsilon \rightarrow 0} \frac{1}{\varepsilon} \operatorname{Card}\left\{x \in r_{\varepsilon}(\tau), h_{\tau}(x)=a\right\}$.

But, by construction, we have, for every $\mathbf{t} \in \mathbb{T}$ and every $\varepsilon>0$,

$\operatorname{Card}\left(\left\{x \in r_{\varepsilon}(\mathbf{t}), h_{\mathbf{t}}(x)=a-\varepsilon\right\}\right)=\operatorname{Card}\left(\left\{x \in r_{\varepsilon}(\mathcal{R}(\mathbf{t})), h_{\mathcal{R}(\mathbf{t})}(x)=H(\mathbf{t})-a\right\}\right)$.

Therefore, we have that for every $a>0, \mathbb{N}^{(\theta)}$-a.e., $\ell_{a}(\tau)=\ell_{H(\tau)-a}(\mathcal{R}(\tau))$. Then, consider the continuous version of the local time to conclude.

3.4. Extension to a forest. For $\theta \geq 0$, we define the Brownian forest as the forest $\mathcal{F}=\left(\left(h_{i}, \tau_{i}\right), i \in I\right)$ where $\sum_{i \in I} \bar{\delta}_{h_{i}, \tau_{i}}$ is a Poisson point measure on $\mathbb{R} \times \mathbb{T}$ with intensity $2 \beta d h \mathbb{N}^{(\theta)}[d \tau]$ and we denote by $\mathbb{P}^{(\theta)}$ its distribution.

Remark 3.11. This Brownian forest can be viewed as the genealogical tree of a stationary continuous-state branching process (associated with the branching mechanism $\psi_{\theta}$ defined in (2.5)), see Chen and Delmas (2012). To be more precise, for every $i \in I$ let $\left(\ell_{a}^{(i)}\right)_{a \geq 0}$ be the local time measures of the tree $\tau_{i}$. For every $t \in \mathbb{R}$, we define the size $Z_{t}$ of the population at time $t$ by

$$
Z_{t}=\sum_{i \in I} \ell_{t-h_{i}}^{(i)}\left(\tau_{i}\right),
$$

where we recall that the local time $\ell_{a}(\tau)$ of the CRT $\tau$ is zero for $a \notin[0, H(\tau)]$. For $\theta=0$, we have $Z_{t}=+\infty$ a.s. for every $t \in \mathbb{R}$. For $\theta>0$, the process $\left(Z_{t}, t \geq 0\right)$ is a stationary Feller diffusion, solution of the SDE

$$
d Z_{t}=\sqrt{2 \beta Z_{t}} d B_{t}+2 \beta\left(1-\theta Z_{t}\right) d t .
$$

A forest $\mathbf{f}=\left(\left(h_{i}, \mathbf{t}_{i}\right), i \in I\right)$ is said to be height-regular if:

- for every $i \in I, \mathbf{t}_{i} \in \mathbb{T}_{0}$

- for every $i, j \in I$, if $i \neq j$, then $h_{i} \neq h_{j}$ and $h_{i}+H\left(\mathbf{t}_{i}\right) \neq h_{j}+H\left(\mathbf{t}_{j}\right)$.

We define the reverse of a height-regular forest $\mathbf{f}=\left(\left(h_{i}, \mathbf{t}_{i}\right), i \in I\right)$ as the forest

$$
\mathcal{R}(\mathbf{f})=\left(\left(-h_{i}-H\left(\mathbf{t}_{i}\right), \mathcal{R}\left(\mathbf{t}_{i}\right)\right), i \in I\right) .
$$

Lemma 3.12. Let $\theta \geq 0$. Let $\left(\left(h_{i}, \tau_{i}\right), i \in I\right)$ be a Brownian forest under $\mathbb{P}^{(\theta)}$. Then the point process

$$
\sum_{i \in I} \delta_{\left(-h_{i}-H\left(\tau_{i}\right), \tau_{i}\right)}(d h, d \mathbf{t})
$$

is a Poisson point process on $\mathbb{R} \times \mathbb{T}$ with intensity $2 \beta d h \mathbb{N}^{(\theta)}[d \mathbf{t}]$. 
Proof: The proof is similar to the one of Lemma 3.8. For every non-negative measurable function $\varphi$ on $\mathbb{R} \times \mathbb{T}$, we have, denoting $\mathbb{E}^{(\theta)}$ the expectation under $\mathbb{P}^{(\theta)}$,

$$
\begin{aligned}
\mathbb{E}^{(\theta)}\left[\mathrm{e}^{-\sum_{i \in I} \varphi\left(-h_{i}-H\left(\tau_{i}\right), \tau_{i}\right)}\right] & =\exp \left(-\int_{-\infty}^{+\infty} 2 \beta d h \mathbb{N}^{(\theta)}\left[1-\mathrm{e}^{-\varphi(-h-H(\tau), \tau)}\right]\right) \\
& =\exp \left(-2 \beta \mathbb{N}^{(\theta)}\left[\int_{-\infty}^{+\infty}\left(1-\mathrm{e}^{-\varphi(-h-H(\tau), \tau)}\right) d h\right]\right) \\
& =\exp \left(-2 \beta \mathbb{N}^{(\theta)}\left[\int_{-\infty}^{+\infty}\left(1-\mathrm{e}^{-\varphi(v, \tau)}\right) d v\right]\right),
\end{aligned}
$$

by an obvious change of variables, which yields the result.

We deduce from Lemma 3.12, Lemma 3.7 and Theorem 3.9 the following corollary.

Corollary 3.13. Let $\theta \geq 0$. Let $\mathcal{F}$ be a Brownian forest under $\mathbb{P}^{(\theta)}$. Then $\mathcal{F}$ is a.s. height regular and the reversed forest $\mathcal{R}(\mathcal{F})$ is distributed as $\mathcal{F}$.

Remark 3.14. Recall notation for forests introduced at the end of Section 2.5. For every real numbers $s<t$, and every forest $\mathcal{F}$, we set

$$
M_{s}^{t}(\mathcal{F})=\operatorname{Card}\left(\left\{x \in \mathcal{F}_{\mathbb{R}}, h_{\mathcal{F}}(x)=s \text { and } H\left(\mathbf{t}_{x}\right) \geq t-s\right\}\right)
$$

the number of vertices of $\mathcal{F}$ at height $s$ that have descendants at time $t$ (excluding the infinite spine). Corollary 3.13 allows to straightforward recover (and understand) Theorem 4.3 from Bi and Delmas (2016) that states that, under $\mathbb{P}^{(\theta)}$ for any $\theta>0$, the processes $\left(M_{s}^{s+r}, s \in \mathbb{R}, r \geq 0\right)$ and $\left(M_{s-r}^{s}, s \in \mathbb{R}, r \geq 0\right)$ are equally distributed. Indeed, to recover this result, it is enough to notice that a.s.:

$$
\left(M_{s}^{s+r}(\mathcal{F}), s \in \mathbb{R}, r \geq 0\right)=\left(M_{s-r}^{s}(\mathcal{R}(\mathcal{F})), s \in \mathbb{R}, r \geq 0\right) .
$$

Remark 3.15. Following Section 7.4 in Athreya et al. (2017), the stationary process $\left(Z_{t}, t \geq 0\right)$ can also be represented as the local time process of $W=\left(W_{t}, t \in \mathbb{R}\right)$ defined by:

$$
W_{t}= \begin{cases}B_{t}^{(\theta)}-2 I_{t}^{(\theta)} & \text { for } t \geq 0 \\ B_{t}^{\prime(\theta)}+2 I_{t}^{\prime(\theta)} & \text { for } t<0\end{cases}
$$

where $B^{(\theta)}=\left(B_{t}^{(\theta)}, t \geq 0\right)$ is a Brownian motion with drift defined by (2.2) and $I_{t}^{(\theta)}$ its infimum defined by $(2.3)$, and $\left(\left(B_{t}^{\prime(\theta)}, I_{t}^{\prime(\theta)}\right), t \leq 0\right)$ is independent of $B^{(\theta)}$ and distributed as $\left(\left(B_{-t}^{(\theta)}, I_{-t}^{(\theta)}\right), t \leq 0\right)$. Then, for every $s \in \mathbb{R}$ and $r>0$, the quantity $M_{s}^{s+r}$ can be expressed as

$$
\begin{array}{r}
M_{s}^{s+r}(W)=\operatorname{Card}\left\{t \in \mathbb{R} ; W_{t}=s \quad \text { and } \exists t^{\prime}>t, W_{t^{\prime}}=s+r\right. \\
\text { and } \left.\inf _{u \in\left(t, t^{\prime}\right)} W_{u}>s\right\}
\end{array}
$$

or equivalently, the number of excursions of $W$ above level $s$ that reach level $s+r$.

Then if we set $\widetilde{W}_{t}=-W_{-t}$, it is easy to see that for every $s \in \mathbb{R}$ and $r>0$, $M_{s}^{s+r}(W)=M_{s-r}^{s}(\widetilde{W})$ which also gives Theorem 4.3 of Bi and Delmas (2016) as $W$ and $\widetilde{W}$ are equally distributed. 
One could therefore ask if the tree coded by any process $W$ that satisfies the equality in distribution $W \stackrel{(d)}{=} \widetilde{W}$ also statisfies the reversal property of Corollary 3.13 (which does not correspond to the transformation $W \longmapsto \widetilde{W}$ on the contour process as remarked after the proof of Theorem 3.9).

\section{References}

R. Abraham and J.-F. Delmas. Williams' decomposition of the Lévy continuum random tree and simultaneous extinction probability for populations with neutral mutations. Stochastic Process. Appl. 119 (4), 1124-1143 (2009). MR2508567.

R. Abraham and J.-F. Delmas. A continuum-tree-valued Markov process. Ann. Probab. 40 (3), 1167-1211 (2012). MR2962090.

R. Abraham, J.-F. Delmas and P. Hoscheit. A note on the Gromov-HausdorffProkhorov distance between (locally) compact metric measure spaces. Electron. J. Probab. 18, no. 14, 21 (2013). MR3035742.

D. Aldous. The continuum random tree. I. Ann. Probab. 19 (1), 1-28 (1991). MR1085326.

S. Athreya, W. Löhr and A. Winter. Invariance principle for variable speed random walks on trees. Ann. Probab. 45 (2), 625-667 (2017). MR3630284.

H. Bi and J.-F. Delmas. Total length of the genealogical tree for quadratic stationary continuous-state branching processes. Ann. Inst. Henri Poincaré Probab. Stat. 52 (3), 1321-1350 (2016). MR3531711.

D. Burago, Y. Burago and S. Ivanov. A course in metric geometry, volume 33 of Graduate Studies in Mathematics. American Mathematical Society, Providence, RI (2001). ISBN 0-8218-2129-6. MR1835418.

Y.-T. Chen and J.-F. Delmas. Smaller population size at the MRCA time for stationary branching processes. Ann. Probab. 40 (5), 2034-2068 (2012). MR3025710.

M. Dávila Felipe and A. Lambert. Time reversal dualities for some random forests. ALEA Lat. Am. J. Probab. Math. Stat. 12 (1), 399-426 (2015). MR3368964.

A. Dress, V. Moulton and W. Terhalle. T-theory: an overview. European J. Combin. 17 (2-3), 161-175 (1996). MR1379369.

T. Duquesne. The coding of compact real trees by real valued functions. ArXiv Mathematics e-prints (2006). arXiv: math/0604106.

T. Duquesne and J.-F. Le Gall. Random trees, Lévy processes and spatial branching processes. Astérisque (281), vi+147 (2002). MR1954248.

T. Duquesne and J.-F. Le Gall. Probabilistic and fractal aspects of Lévy trees. Probab. Theory Related Fields 131 (4), 553-603 (2005). MR2147221.

S. N. Evans. Probability and real trees, volume 1920 of Lecture Notes in Mathematics. Springer, Berlin (2008). ISBN 978-3-540-74797-0. MR2351587.

S. N. Evans, J. Pitman and A. Winter. Rayleigh processes, real trees, and root growth with re-grafting. Probab. Theory Related Fields 134 (1), 81-126 (2006). MR2221786.

M. Gromov. Metric structures for Riemannian and non-Riemannian spaces, volume 152 of Progress in Mathematics. Birkhäuser Boston, Inc., Boston, MA (1999). ISBN 0-8176-3898-9. MR1699320.

A. Lambert. Quasi-stationary distributions and the continuous-state branching process conditioned to be never extinct. Electron. J. Probab. 12, no. 14, 420-446 (2007). MR2299923. 
J.-F. Le Gall and Y. Le Jan. Branching processes in Lévy processes: the exploration process. Ann. Probab. 26 (1), 213-252 (1998). MR1617047.

J. Neveu and J. Pitman. The branching process in a Brownian excursion. In Séminaire de Probabilités, XXIII, volume 1372 of Lecture Notes in Math., pages 248-257. Springer, Berlin (1989a). MR1022915.

J. Neveu and J. Pitman. Renewal property of the extrema and tree property of the excursion of a one-dimensional Brownian motion. In Séminaire de Probabilités, XXIII, volume 1372 of Lecture Notes in Math., pages 239-247. Springer, Berlin (1989b). MR1022914.

S. Roelly-Coppoletta and A. Rouault. Processus de Dawson-Watanabe conditionné par le futur lointain. C. R. Acad. Sci. Paris Sér. I Math. 309 (14), 867-872 (1989). MR1055211. 